\title{
The Jordan Curve Theorem and an Unpublished Manuscript by Max Dehn
}

\author{
H. Guggenheimer \\ Communicated by C. TRUESDELL
}

1.

The JoRdan Curve Theorem is the basis of a correct development of the CAUCHY-RIEMANN approach to the theory of functions of a complex argument. CAUCHY himself discovered his integral formula [1] by formally adding real integrals; in his coherent development of function theory [2] he simply speaks of "points renfermés dans une certaine aire qu'enveloppe un certain contour". RIEMANN's thesis [3], contemporary with [1], contains a correct definition of simply and multiply connected domains but neither there nor in the theory of ABELIAN functions [4], published one year after the appearance of [2], is there any attempt to prove that certain curves (e.g., the circle) in fact form the boundary of simply connected domains. The geometric deficiencies seem to be one of the main reasons that moved WeIERSTRASS to develop function theory on an arithmetic basis; the canonical exposition of WEIERSTRASs's theory [5] manages to avoid the use of any integrals with the exception of integrals on real intervals and for the computation of the periods of elliptic functions on pp. 369-402. Of the textbooks of function theory written before JoRDAN's Cours d'analyse, only C. Neumann [6] and J. ThOmaE [7] seem to have noted the topological problem. NEumanN's book is important for the development of geometric function theory since for the first time it introduces the representation of complex numbers on the RiEmanN sphere. The author notes that this is “ein Gedanke, der mir aus Riemann's Vorlesungen durch mündliche Ueberlieferung zu Ohren kam..." ([6], Vorwort zur ersten Auflage, p. v of the second edition). For the treatment of curves, the book is on the level of CAUCHY. As the author notes ([6], Vorwort zur zweiten Auflage, p. viii), "Absichtlich habe ich indessen in dieser Beziehung die Theorie in derjenigen Form, in welcher sie von CAUCHY und RIEMANN gegeben ist, zu conserviren gesucht. ... Ueberhaupt dürfte es ja bei der Darlegung einer mathematischen Theorie weniger auf eine durchweg strenge Darstellung, als vielmehr darauf ankommen, daß die angegebenen Methoden die zur strengen Darstellung erforderlichen Mittel gewähren." ThomaE's book [7] (his third text of function theory) gives RiemanN's definition and (p. 5) declares it intuitively clear that the circle and its topological equivalents are boundaries of simply connected domains. (THOMAE is a co-discoverer of the DARBOUX integral and author of some early papers on set theory applied to analysis.) 
The recognition of the central role of the curve theorem and the problem of its proof is the lasting contribution of C. JORDAN [8]. JORDAN does not prove the theorem. He accepts as obvious (Sec. 99, p. 94, all quotations from the second edition) that a simple closed polygon separates the plane into two regions in which two points can be joined by a polygonal arc without crossing the polygon but that any polygonal arc joining two points in distinct regions must cross the polygon (Sec. 103, p. 99). He proves that for sufficiently small $\varepsilon>0$ one can find polygons $S_{\varepsilon}, S_{\varepsilon}^{\prime}$ inscribed and circumscribed to the curve $C$ so that the distance of points of $S_{\varepsilon}$ and $S_{\varepsilon}^{\prime}$ from a point of $C$ is $\leqq \varepsilon$. A point in the interior of $C$ if it is in the interior of some $S_{\varepsilon}$ and in the exterior of $C$ if it is in the exterior of some $S_{\varepsilon}^{\prime}$. Therefore, $C$ divides the plane into interior, exterior, and $C$ itself. In addition, JORDAN shows that the bounded domain defined by $C$ is simply connected in the sense of RIEMANN (Sec. 104, p. 99). In the absence of a clear formulation of the notions of compactness and the HeINe-Borel theorem, the first step (lines 10-12 on p. 93, Sec. 98) of the proof of the existence of $S_{\varepsilon}$ and $S_{\varepsilon}^{\prime}$ is not convincing; what is missing is a proof that for a JoRDAN curve $x(t), 0 \leqq t \leqq 1$, and $\delta>0$ there is an $\varepsilon_{0}$ such that segments $x\left(t_{i}\right) x\left(t_{i+1}\right), x\left(t_{j}\right) x\left(t_{j+1}\right)$ of a polygon of vertices $x\left(t_{0}\right), \ldots, x\left(t_{N}\right)$ cannot intersect if $t_{k+1}-t_{k}<\varepsilon \leqq \varepsilon_{0}$ and $\left|x\left(t_{j+1}\right)-x\left(t_{i}\right)\right|<\delta$. This gap is filled in [22]. The proof is not helped by JoRDAN's use of $t_{i}$ for both $t_{i}$ and $x\left(t_{i}\right)$.

2.

The importance of the JoRDan Curve Theorem was recognized quickly after the publication of the second edition of the Cours d'Analyse in which the theorem and its proof were given prominent display in the first chapter. The difficulty of the proof generated an interesting literature. The literature up to $1910 / 11$ is surveyed in the following paragraphs.

The first attempt to prove the complete JORDAN theorem is contained in the paper of 1896 of SCHOENFLIES [9], his first paper on the subject. The work is based on PASCH's analysis [10] of the separation of the plane by straight segments and triangles and is as correct as it can be in the absence of an axiomatic basis of topology. The proof is sketchy in places but the gaps are not serious. SCHOENFLIES's proof is valid for piecewise $C^{\prime}$-curves of finite order, i.e., curves intersected by any straight line in a finite number of points only. The main idea is that an interior point is characterized by the property that any ray through the point meets the curve (by convenient computation of multiplicities) at an odd number of points. The finite order is used again in the proof that any two interior points can be joined by a polygonal arc. SCHOENFLIES achieves the equivalent of a triangulation by dividing the interior into a finite number of simple domains. He fixes a direction and draws the parallels through the cusps of the curve and all tangents in the given direction. Since a JORDAN arc of finite order must be of finite class, one obtains a finite number of domains bounded by arcs of the curve and parallel straight segments; these domains are, in modern language, arcwise connected and simply connected.

The proof in DE LA VALLÉE Poussin's Cours d'Analyse [11] follows JORDAN in accepting the polygon theorem; the proof is sketchy in the first two editions. 
The first attempt at an axiomatic proof of the JORDAN Theorem for polygons is in Veblen's thesis [12]. The main object of the thesis is a revision of the axiom system of PASCH [10] by the addition of existence axioms in the style of Hilber t and the removal of redundant axioms. The second part of the thesis is devoted to proofs based on the axiom system; the JORDAN Theorem appears among the consequences of PASCH's axioms of order. VeBLEN's proof depends on two lemmata:

1. If a side of a polygon $q$ intersects a side of a polygon $p_{n}$ in a single point $O$ not a multiple point $p_{n}$ or $q$ then $p_{n}$ and $q$, whether simple or not, have at least one other point in common.

2. Every point $P$ of the boundary of a simple polygon $p_{n}$ is accessible from every point $O$ not on the boundary of $p_{n}$, by a broken polygonal line joining $O$ to $P$ and not meeting the boundary of the polygon in any point than $P$.

Lemma 1 implies that the polygon divides the plane into at least two regions and lemma 2 shows that there can be no more than two regions. As pointed out by LENNES [24] and H. HAHN [23], the proof of the lemmata presupposes a proof that a polygon can be triangulated, which is missing. In the absence of a triangulation, VeBLEN's proof is valid only for convex polygons. A later section of [12] deals with the separation of space by a simple polyhedron.

Another proof of the JORDAN Theorem for curves is in the Harvard thesis of L. D. AMES [12, 14] and is reproduced in OsGoOD's Funktionentheorie [15]. The proof is based on the KRONECKER characteristic (Umlaufzahl). AMEs's restriction to piecewise $C^{\prime}$ curves is unnecessary. The existence of at least two domains (of Umlaufzahl 0 and \pm 1 ) is immediate. The proof that not more than two domains exist is given only for curves that are finite unions of arcs schlicht over a coordinate axis.

The proof of BLISs as sketched in [16] is valid for curves of finite order. The domains obtained by ScHOENFLIES [9] are characterized by the sign of conveniently chosen functions if the fixed direction is parallel to one of the coordinate axes.

VEBLEN's proof [17] of the JoRdAN Curve Theorem on the basis of E.H. Moore's topological axioms for General Analysis is based on the ideas of [12] and open to the same objections. In addition, VEBLEN's proof as well as SCHOENFLIES's later investigations $[18,19]$ on the topology of JORDAN curves are open to BROUWER's objection [20] that an arc in the plane can be the common boundary of more than two domains.

SCHOENFLIES's investigations are based on the JORDAN Theorem for polygons which follows easily from [9]. The important paper is [19]; the first part of the paper is devoted to the proof that the points of a JORDAN curve are accessible from the interior and the exterior, in the sense of VeBLEN's lemma 2, by JORDAN arcs. This follows from the corresponding result for JoRDAN polygons. The second part contains formulation and proof of the statement known as the SCHOENFLIES Theorem: Every homeomorphism of a JORDAN curve and a circle can be extended to an homeomorphism between the interior of the curve and a circular disc. SCHOENFLIES actually proves the theorem for a JoRDAN polygon and a square. The idea is simple: One chooses an interior point $O$ and connects 
$O$ to all points of the polygon by non-intersecting polygonal lines. The polygonal lines are then mapped onto the rays leading from the center of the square to the square. The technical difficulties of the construction are considerable. The proof is adapted in [21] with very minor changes to yield directly the SCHOENFLIES Theorem for JORDAN curves: SCHOENFLIES's arguments in the plane case are correct even though the examples of ANTOINE and AlEXANDER show that in space the inference from polyhedra to general surfaces is false.

The proof of W. H. \& G. CH. Young ([22], pp. 224-228) proceeds in two steps. First it is shown that a JORDAN curve which is a union of finitely many circular arcs divides the plane into at least two domains. Then the HEINEBOREL Theorem is used to cover a given JORDAN curve with a finite number of circular discs of radius $<\varepsilon$ to get a domain bounded by two JORDAN curves composed of circular arcs. For $\varepsilon \rightarrow 0$, it follows from CANTOR's theorem on the intersection of compact sets that the points of the given curve are accessible from interior and exterior. Therefore, the number of domains cannot be $>2$.

The paper of 1908 of $\mathrm{H}$. HAHN [23] is a complete axiomatic proof of the JoRdan Curve Theorem for polygons, based on the Pasch-Veblen system. The paper is important because it develops systematically the properties of the order relation which today are reproduced in all books of axiomatic elementary geometry. The enumeration of these properties is incomplete in the book of PASCH and rudimentary in the "Grundlagen" of HilberT. HAHN proves the JORDAN Theorem first for unbounded angles, then for polygons formed by segments on the legs of an angle and connecting polygonal lines, and finally for general polygons by reduction to the case of polygons in angles.

The papers by N. J. LeNNES $[24,25]$ are similarly important. The starting point of LENNES is the observation that HILBERT's axiomatic theory of area and volume depends on the existence of triangulations of polygons, or simplicial decompositions of polyhedra. No proof of the existence of such a decomposition is given in the "Grundlagen der Geometrie" or subsequent publications of the HILBERT school. $\S 1$ of [24] contains a study of the separation of the plane by angles and triangles. $\S 2$ contains a proof of the JoRDAN Curve Theorem in the form: A simple polygon separates the remaining points of the plane into two open connected and arcwise connected sets such that every broken line connecting points not in the same set meets the polygon. The proof starts with the definition of interior points following [9]: the definition is shown to be independent of the ray chosen by an argument found also in [23]. Finally it is shown by an original construction that two points in the same set (interior or exterior) can be connected by a sequence of triangles separated from the polygon. This proves openness and connectedness at the same time. $\S 3$ contains the proof that a polygon can be decomposed into triangles using only the vertices of the polygon as vertices of triangles. The main lemma states that each polygon has at least one projecting vertex, i.e., a vertex for which there exists a line intersecting the polygon at a point of each of the two edges meeting at the vertex and at no other point. The proof of the lemma does not use the JoRDAN Curve Theorem. The triangulation is obtained by reduction: the projecting vertex either is a vertex of a triangle which does not contain a vertex of the polygon in its interior or it is the endpoint of a diagonal splitting 
the polygon into two JORDAN polygons with fewer vertices. $\S 4$ gives a new proof of the JoRDAN Curve Theorem based on the triangulation: the interior of the curve is the union of the interiors of the triangles of the triangulation and the open diagonals. $\S 5$ takes up the definition of polyhedra and related sets of solid geometry. $\S 6$ proves the space analog of the JoRDAN Theorem for simple polyhedra based on the counting definition of [9]. $\S 7$ contains the example of a polyhedron with 7 vertices which does not admit a simplicial decomposition using only the vertices of the polyhedron as vertices of the tetrahedra. The same section contains a proof that every JORDAN polyhedron can be decomposed into convex polyhedra and that every convex polyhedron admits a simplicial decomposition. The problems touched upon in this section reappear in higher dimensional algebraic topology as the problems of the relations of cell complexes, simplicial complexes, and the Hauptvermutung. The final $\S 8$ contains an axiomatic study of the definition of polyhedron.

The paper [25] continues [24] for JoRDAN curves, using only topological properties that can be formulated without recourse to metrical notions. The topology is generated by the open half-planes of a geometry satisfying the axioms of incidence and order of PASCH-VeBLEN. (Such a topology is always completely regular and is metrizable by an ordered ABELIAN group [26].) LENNES first studies JoRDan curves which are unions of a countable set of straight segments. Then follows studies of the meaning of "curve" and "boundary". LENNES avoids becoming vulnerable to BROUWER's objections to the work of SCHOENFLIES by admitting only domains whose boundary points are arcwise accessible from the interior; the discussion ends with the characterization of the JORDAN curve as the boundary of a bounded domain accessible both from the interior and the exterior. That the hypothesis of accessibility is needed is illustrated by domains bounded by several spirals (note on bottom of p. 313) similar to BROUWER's examples of continua forming the simultaneous boundary of many domains. LENNES lost the priority in these developments to BRouWER by publishing only in 1911 the paper read to the American Mathematical Society in December of 1905. After these preliminaries, the proof of the JoRDAN Curve Theorem reduces to an application of the HEINE-BOREL Theorem. The remainder of the paper is devoted to a study of the Ascoli Theorem in questions of approximation of curves by simple arcs.

BRouWER's proof [27] of the JoRDAN Curve Theorem is probably the direct topological proof that is most clear and unobjectionable. Starting from some basic theorems on continua, BROUWER proves that (a) an arc between two disjoint continua defines only one domain, (b) two disjoint ares between two disjoint continua together with the continua determine two domains and, (c) each subarc of a JORDAN curve is accessible from every domain it bounds. This implies that a JORDAN curve is the boundary of every domain it bounds and determines neither less nor more than two domains.

3.

The DeHN Archives, administered by Professor Wilhelm Magnus, contain a manuscript by MAX DEHN entitled: "Beweis des Satzes, dass jedes geradlinige 
geschlossene Polygon ohne Doppelpunkte 'die Ebene in zwei Teile teilt'”. On the basis of DeHN's indications, MAGNUS in [28] p. 28 dates the manuscript to 1899 when DeHN was working on his thesis (1900) under HilberT. The date of the manuscript given by MAGNUS is consistent with the spelling, which antedates the unification edict of 1903 . It is a mystery why DEHN never published the paper, which was ahead of its time for many years. In addition to a proof of the JORDAN Curve Theorem, the manuscript contains a proof of the SCHOENFLIES Theorem ( 7 years before SCHOENFLIES's discovery) which is elementary and very simple; the idea of the proof is very close to one given by R. H. BiNG in 1960 [30]. The proof is based on an useful lemma quoted in [28] which was rediscovered only in 1975 [29]. The manuscript also contains a proof of LENNES's theorem that a polygon can be triangulated by diagonals. Since, as LENNES noted, this is a necessary step in HILBERT's theory of area, it is the more remarkable that the proof, found in the year the "Grundlagen der Geometrie" were first published, never was published.

The manuscript is based on HILBERT's axioms of incidence and order and avoids all notions and methods that cannot be derived by these axioms. The first part deals with the plane and contains a proof of the JORDAN Theorem for polygons based on SCHOENFLIES's definition [9] of interior points. The treatment parallels that of [23] and [24]; it is superior to the treatment of [24] because of the truly exhaustive consideration of cases of special position. The codification of properties of incidence and order given in [23] could be used today to simplify DEHN's proof. The proof that interior and exterior are arcwise connected precedes the triangulation theorem and is therefore exceedingly lengthy and difficult.

A vertex $P_{i}$ of a JoRDAn polygon $P_{0} \ldots P_{N} P_{0}$ is a convex corner if there are an $A$ on $P_{i-1} P_{i}$ and a $B$ on $P_{i} P_{i+1}$ such that the open segment $A B$ is in the interior of the polygon. The second topic of the paper is a proof that a polygon has at least three convex corners. Since the axioms do not imply the theorems of Desargues and PAPPUS, one has to study the properties of the convex hull without using linearity. A convex corner $P_{i}$ is called an ear in [29] if no point of the polygon is in the open triangle $P_{i-1} P_{i} P_{i+1}$ or on the open segment $P_{i-1} P_{i+1}$. The proof of the existence of at least two ears for every JORDAN polygon given in [29] uses metric notions. DEHN first notes that a convex corner $P_{i}$ either is an ear or there exists a vertex $P_{j}$ in $P_{i-1} P_{i} P_{i+1}$ so that one halfplane of $P_{i+1} P_{j}$ contains only the vertex $P_{i}$ while all other vertices are in the closed complementary halfplane. (A similar argument is used in [24], $\S 3$.) In the second case, $P_{i} P_{j}$ is a diagonal contained in the interior of the polygon which divides the polygon into two JORDAN polygons with fewer vertices. After a finite number of steps, one obtains a triangulation of the polygon and in the final step in each partial polygon one obtains an ear: every JORDAN polygon has at least two ears (the "three" in [28] is a misprint from the three convex corners). BING [30, p. 32] proves only that every JORDAN polygon has vertices $P_{i-1} P_{i} P_{i+1}$ so that no other vertex of the polygon is in the interior of the triangle $P_{i-1} P_{i} P_{i+1}$ or on the open segment $P_{i-1} P_{i+1}$; the proof can be adapted to DeHN's axiomatic framework.

Next comes a construction of an order preserving map of a segment $A C$ 
onto a subsegment $B C$ : Choose $\mathrm{O}_{2}$ in an halfplane of $A C$ and points $E, D$ such that the order relations $\left(\mathrm{AO}_{2} \mathrm{D}\right)$ and $\left(\mathrm{BO}_{2} E\right)$ hold. Then, by PASCH's axiom, $C O_{2}$ intersects $B D$ at $O_{1}$ and $\left(B O_{1} D\right)$ holds. Put $Y=X O_{2} \cap E D, F(X)=A C \cap Y O_{1}$ for $X$ on $A C$. The map $X \rightarrow F(X)$ is order preserving by repeated application of PASCH's axiom. This is now used for a proof of the SCHOENFLIES Theorem: For an ear $P_{i}$ we may choose $Q$ so that $P_{i-1} Q P_{i+1}$ is in the interior of the polygon and $R$ so that $P_{i-1} R P_{i+1}$ is in the exterior of the polygon. Let a ray through $Q$ intersect $P_{i-1} P_{i+1}$ at $X, P_{i-1} P_{i} P_{i+1}$ at $Y$ and $P_{i-1} R P_{i+1}$ at $Z$. By the previous construction we can obtain an order preserving one-to-one map of the ray onto itself that is the identity outside $Q Z$, maps $Q Y$ onto $Q X$ and $Y Z$ onto $X Z$. Hence we obtain a one-to-one map of the plane onto itself which maps the interior of the polygon onto the interior of polygon with one vertex less. After a finite number of steps, the polygon is mapped onto a triangle. (The paper is not concerned with continuity but a little care in choosing the maps on the single rays will make the map of the plane continuous in the topology generated by the open half-planes.) The same basic idea of the proof of the SCHOENFLIES theorem is given in [30], p. 31-33. There the rays are mapped onto themselves by piecewise linear maps which cannot be defined using only axioms of order and incidence. The construction yields the following result, which I cannot find in the published literature: The SCHOENFLIES Theorem is valid in a plane geometry based on HILBERT's axioms of order and incidence only.

The last part of the paper contains a proof of the JORDAN Theorem for polyhedra roughly parallel to [24] $\$ 6$ with all details filled in.

\section{Bibliography}

1. Cauchy, A.L., Sur les diverses relations qu'existent entre les résidus des fonctions et les intégrales définies. Exercices d'Analyse 2 (1831) =Oeuvres II 6, 124-145.

2. Cauchy, A.L., Sur la théorie des fonctions, C.R. Acad. Sci. Paris 43 (1856) 69=Oeuvres I $12,235$.

3. RIEMANN, B., Grundlagen für eine allgemeine Theorie der Functionen einer veränderlichen complexen Grösse. Dissertation Göttingen 1831 = Gesammelte mathematische Werke 3-45.

4. Riemann, B., Theorie der abelschen Functionen. J.f.d. reine u. angew. Math. 54 (1857) 115-169= Gesammelte mathematische Werke 88-142.

5. Biermann, O., Theorie der analytischen Functionen, Teubner, Leipzig 1887.

6. NeumanN, C., Vorlesungen über Riemann's Theorie der Abel'schen Integrale. Teubner, Leipzig 1865, 2. Aufl. 1884.

7. ThOMAE, J., Theorie der Functionen eines complexen Arguments, Halle 1891.

8. Jordan, C., Cours d'Analyse, Gauthier-Villars, Paris. 1'éd., vol. 3 (1887) p. 587-594, $2^{e}$ éd. vol. 1 (1893) 90-99.

9. Schoenflies, A., Ueber einen Satz aus der Analysis situs. Nachr. Ges. Wiss. Göttingen, Math. Phys. K1. 1896, 79-89.

10. Рasch, M., Vorlesungen über neuere Geometrie, Teubner, Leipzig 1882

11. De la Vallée Poussin, C., Cours d'analyse infinitésimale, Libraire universitaire Louvain, Gauthier-Villars Paris, $1^{\circ}$ éd. 1903, vol. 1, p. 308; $3^{\mathrm{e}}$ éd. 1914, vol. 1, 374-379.

12. Veblen, O., A System of Axioms for Geometry, TAMS 5 (1904) 343-380.

13. Ames, L.D., On the Theorem of Analysis Situs Relating to the Division of the Plane or of Space by a Closed Curve of Surface, BAMS 10 (1904) 301-305.

14. Ames, L.D., An Arithmetic Treatment of Some Problems in Analysis Situs, Amer. J. Math. 27 (1905) 345-358. 
15. OsGood, W.F., Allgemeine Funktionentheorie I, Teubner, Leipzig 1906.

16. Bliss, G.A., The Exterior and Interior of a Plane Curve, BAMS 10 (1904) 398-404.

17. Veblen, O., Theory of Plane Curves in Non-Metrical Analysis Situs, TAMS 6 (1905) 83-98.

18. Schoenflies, A., Beiträge zur Theorie der Punktmengen, II. Math. Ann. 59 (1904) 129-160.

19. Schoenflies, A., Beiträge zur Theorie der Punktmengen III. Math. Ann. 62 (1906) 286-328.

20. Brouwer, L.E.J., Zur Analysis Situs. Math. Ann. 68 (1910) $422-434$.

21. Hall, D.W., \& Spenger, G.L. II, Elementary Topology. Wiley, New York, 1955.

22. Young, W.H., \& Young, G.C., The Theory of Sets of Points. Cambridge 1906 (reprinted, Chelsea, New York 1972).

23. Hahn, H., Über die Anordnungssätze der Geometrie. Monatsh. Math. Phys. 19 (1908) 289-303.

24. Lennes, N.J., Theorems on the Simple Finite Polygon and Polyhedron, Amer. J. Math. 33 (1911) 37-62, read to the AMS in April, 1903.

25. Lennes, N.J., Curves in Non-Metrical Analysis Situs with an Application in the Calculus of Variations, Amer. J. Math. 33 (1911) 287-326, read to the AMS in December, 1905.

26. GugGenheimer, H., Méthodes topologiques en fondements de la géometrie, Bull. Res. Counc. Israel 6A (1957) 103-114.

27. Brouwer, L.E.J., Beweis des Jordanschen Kurvensatzes, Math. Ann. 69 (1910) 169-175.

28. Magnus, W., Noneuclidean Tesselations and Their Groups, Academic Press, New York-London, 1974.

29. Meisters, G.H., Polygons Have Ears, Amer. Math. Monthly 82 (1975) 648-651.

30. Bing, R.H., Elementary Point Set Topology. 8th Hubert Ellsworth Slaught Memorial Paper, Amer. Math. Monthly 67 (1960) no. 7, part II.

Polytechnic Institute of New York Brooklyn 